\title{
Obtenção da Imagem de Defeitos em Materiais por Mapeamento Elétrico
}

\author{
Gil R. V. Pinheiro* Victor G. Silva ${ }^{* * *}$ George L. dos Santos* \\ José A. C. P. Gomes ${ }^{* *}$ Jorge L. M. do Amaral* \\ * Departamento de Eletrônica e Telecomunicações, Universidade do \\ Estado do Rio de Janeiro, RJ. \\ ** Departamento de Engenharia de Materiais, Universidade Federal do \\ Rio de Janeiro, RJ. \\ *** CENPES, Petróleo Brasileiro S.A., RJ.
}

E-mails: gilp@uerj.br, vgsilva@petrobras.com.br, georgeleandro_santos@hotmail.com, ponciano@metalmat.ufrj.br, jorge.luis.machado.amaral@gmail.com

\begin{abstract}
This work presents a system proposal for measurement and digital processing of electric potential drop, using a scanning electrode, to get a mapping of surface electric potentials. The processed signal allows to detect the morphology of internal defects such as cracks, corrosion, welding defects and other defects in piping, pressure vessels and others industrial equipments. The proposed technique allowed to infer the electrical image of an internal defect in a stainless steel plate. The technique proposed on this paper offer a real time tool for monitoring and analysis, for the prevention of failure, leakages and avoiding potential incidents in pipes and equipments made of carbon steel, stainless steel, and similar electrical conductive materials, used in installations of petroleum industry, water and sewage treating plants, thermoelectric plants, among others. The proposed solution can be developed to a field equipment to create electrical images based in potential drop.

Resumo: Este trabalho apresenta uma proposta de sistema para a medição e processamento digital de sinais de diferença de potencial elétrico, utilizando um eletrodo de varredura, para obter um mapeamento de potenciais elétricos de superfície. Utilizando técnicas de processamento de sinais, é possível detectar a morfologia de defeitos internos, como trincas, corrosão, defeitos em soldas e falhas em tubulações, vasos de pressão e outros equipamentos industriais. A técnica proposta permitiu inferir a imagem elétrica de um defeito interno numa chapa de aço inox. A técnica apresentada oferece uma ferramenta em tempo real para o monitoramento e análise, prevenção de falhas, de vazamentos e de incidentes em tubulações e equipamentos de aço carbono, aço inoxidável e materiais condutores elétricos similares, utilizados em instalações petrolíferas, de tratamento de água e esgoto, unidades termoelétricas, entre outros. A solução proposta pode resultar num equipamento de campo para criar imagens elétricas baseadas em potential drop.
\end{abstract}

Keywords: Potential drop; corrosion detection; corrosion monitoring; virtual instrumentation; finite element modeling.

Palavras-chaves: Diferença de potencial; deteç̧ão de corrosão; monitoração de corrosão; instrumentação virtual; modelagem por elementos finitos.

\section{INTRODUÇÃO}

A monitoração da corrosão interna constitui uma importante atividade para o gerenciamento da corrosão e da integridade de equipamentos. Através desta atividade é possível entender melhor o processo corrosivo e selecionar o método de controle mais adequado para cada sistema através de diferentes métodos (Roberge, 2007), (Al-Shamari et al., 2014). Dentre os métodos de avaliação do potencial de corrosividade de fluidos estão a obtenção da taxa de corrosão, através de cupons e sondas, análises químicas do fluido e resíduos, dados de PIG instrumentados e histórico de falhas (Yang, 2008), (NITG390, 2013). Uma das etapas relevantes do monitoramento da corrosão interna é a de levantamento de taxas de corrosão, para determinação do potencial de corrosividade do fluido. Geralmente, esta etapa é realizada através da utilização de cupons de perda de massa e sondas de resistência elétrica (RE), que são inseridos no interior do duto ou tubulação. Estes métodos intrusivos necessitam de intervenções periódicas, ou para a troca do cupom de perda de massa para sua posterior avaliação, ou para a substituição do elemento sensor da sonda de resistência elétrica. A periodicidade da troca do cupom e do elemento sensor da sonda RE varia de acordo com a corrosividade do fluido. Esta atividade é considerada crítica em unidades off-shore de produção de petróleo 
(NITG390, 2013). Devido ao elevado risco associado à atividade de troca de cupom e sondas intrusivas, além das dificuldades relacionados à logística, custo e treinamento de pessoal qualificado, os equipamentos não intrusivos vem se tornando mais uma alternativa para a determinação das taxas de corrosão (Silva et al., 2017). A maioria dos equipamentos não intrusivos comerciais que são aplicados para o monitoramento da corrosão interna possuem o princípio físico baseado em ultrassom, porém, alguns equipamentos se baseiam no método do campo elétrico (EFM), também conhecido como potential drop (NITG390, 2013). A técnica de Potential Drop (PD) tem sido usada na indústria para o monitoramento da corrosão interna, através da medição da perda de espessura. Outra aplicação comum desta técnica é a estimativa da profundidade de trincas em dutos, tubulações e equipamentos de processo. Outra aplicação desta técnica é na realização de ensaios de laboratório para avaliar a propagação de trincas. Estes ensaios, geralmente, utilizam o PD para estimar a velocidade de propagação de trincas para a determinação da tenacidade à fratura de um material (Yang, 2008). Apesar de ser uma das técnicas não destrutivas mais antigas para o monitoramento da propagação de trincas e medição de espessura, poucos resultados quantitativos são apresentados na literatura, devido a necessidade de análise matemática ou através de métodos computacionais. Alguns trabalhos, mostram que é possível alcançar uma sensibilidade de detecção razoável utilizando instrumentos de medição específicos e análise dos resultados através de métodos matemáticos e processamento de sinais (Sposito, 2009), (Sposito et al., 2010). Este trabalho tem como objetivo apresentar, através de modelagem em elementos finitos (FEM) e testes em laboratório, a aplicação da técnica de potential drop (PD) na detecção de defeitos internos, explorando novos desenvolvimentos, que ainda podem ser implementados, para tornar esta técnica uma confiável ferramenta de monitoração da corrosão e de propagação de trincas, para futuras aplicações industriais. Para esta finalidade, serão apresentados os princípios e a instrumentação de PD, especificamente desenvolvidos e utilizados, para atender os objetivos deste trabalho. A instrumentação desenvolvida utiliza os conceitos de instrumentação virtual baseada no software LabVIEW ${ }^{\circledR}$, com processamento de sinais feito em Matlab ${ }^{\circledR}$. Também foi realizada a modelagem em FEM com o software COMSOL ${ }^{R}$.

\section{A TÉCNICA DE POTENTIAL DROP}

Numa placa de material condutor de eletricidade, por onde flui uma corrente elétrica, é possível medir diferenças de potenciais (Potential Drop - PD) em sua superfície, que estarão relacionadas às variações nas densidades de corrente no interior da placa, resultantes de defeitos internos no material próximos onde são realizadas as medições de potencial superficial. Utilizando-se este princípio, alguns trabalhos têm sido reportados (Sposito, 2009), (Sposito et al., 2010), mostrando que a técnica PD pode também ser utilizada no monitoramento da corrosão em tubulações metálicas, através da medição da perda de espessura. Em contrapartida, poucos trabalhos apresentam resultados quantitativos utilizando-se PD para a medição da perda de espessura e da geração de imagens, obtidas a partir do processamento dos sinais elétricos medidos,

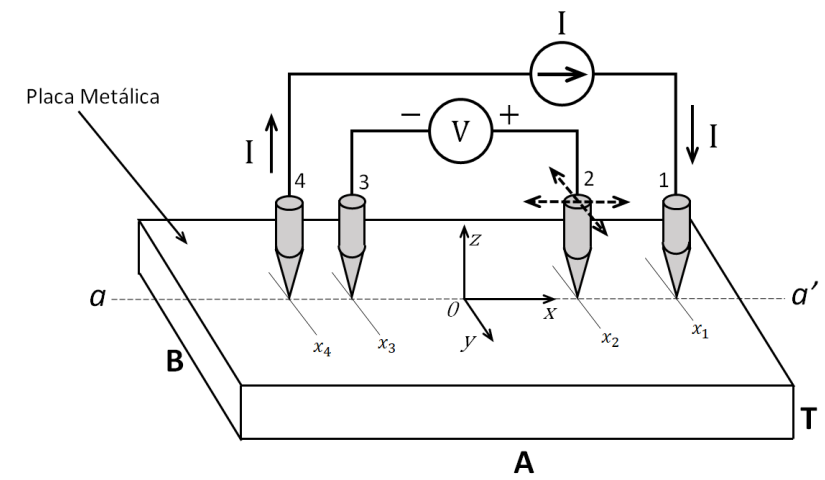

Figura 1. Configuração de eletrodos para medição do PD superficial.

devido à necessidade da análise e processamento de sinais de imagem mais apurados, para se obter este tipo de informação (Sposito, 2009). Para a realização do teste de PD é necessário um arranjo, geralmente, de quatro eletrodos, onde os eletrodos externos são responsáveis pela injeção da corrente elétrica e os internos permitem a medição da diferenças de potencial em vários pontos da superfície de uma peça. O arranjo apresentado na Figura 1 é a configuração mínima para a realização do ensaio de PD. $\mathrm{Na}$ prática, outras configurações têm sido empregadas para que seja possível monitorar uma região de maior dimensão.

\subsection{Injeção de Corrente e Medição de Potencial}

A Figura 1, mostra uma placa metálica, com dimensões: comprimento $=A$, largura $=B$ e espessura $=T$. Na figura, é mostrado o arranjo padrão de 4 eletrodos de pontas finas (1, 2, 3 e 4) alinhados (linha $a a^{\prime}$ ), onde $y=0$ e $z=0$. As coordenadas dos eletrodos são $\left(x_{1}, 0,0\right),\left(x_{2}, 0,0\right),\left(x_{3}, 0,0\right)$ e $\left(x_{4}, 0,0\right)$ respectivamente. A fonte $I$ injeta uma corrente de intensidade $I$ na placa através dos eletrodos $1(+$ da fonte) e 4(- da fonte).

A intensidade de corrente $I$, que atravessa uma área de seção reta $A$ da placa, perpendicular ao eixo $x$ na Figura 1 , está associada à densidade de corrente $\mathbf{J}$, através de (1).

$$
I=\oint_{A} \mathbf{J} d S
$$

Com a corrente $I$ circulando entre os eletrodos e 1 e 4 , podem ser medidas diferenças de potencial na superfície da placa. Através do voltímetro $V$ e dos eletrodos 2 e 3 , cujas posições $x_{2}$ e $x_{3}$ estão localizadas entre os eletrodos 1 e 4 . O eletrodo 2 é móvel, através de motor de passo, podendo medir em várias coordenadas $(x, y, 0)$ na superfície da placa $(z=0)$. O eletrodo 3 é fixo em $\left(x_{3}, 0,0\right)$ e usado como referência nas medições de potencial. A partir dos eletrodos 2 e 3 é possível obter um mapeamento de potenciais $V(x, y, 0)$, referenciado à origem $(0,0,0)$, a partir de $(2)$.

$V(x, y, 0)=V_{2}(x, y, 0)-V_{3}\left(x_{3}, 0,0\right)-V_{R E F}$

Onde:

$V_{R E F}=V_{2}(0,0,0)-V_{3}\left(x_{3}, 0,0\right)$

Os eletrodos 2 e 3 possuem um sistema de molas, de modo a manterem-se em contato permanente com a placa. 
A partir dos potenciais $V(x, y, 0)$, é possível determinar o campo elétrico superficial na placa, através de (3).

$$
\mathbf{E}=\left(E_{x}, E_{y}, 0\right)=-\nabla V=-\left(\frac{\partial}{\partial x}, \frac{\partial}{\partial y}, \frac{\partial}{\partial z}\right) V
$$

O gradiente de potencial superficial, $\nabla V$, pode ser obtido a partir dos potenciais superficiais $V(x, y, 0)$ através de (4).

$$
\begin{aligned}
& \frac{\partial V}{\partial x}(x, y, 0)=\frac{V(x, y, 0)-V(x-\Delta x, y, 0)}{\Delta x} \\
& \frac{\partial V}{\partial y}(x, y, 0)=\frac{V(x, y, 0)-V(x, y-\Delta y, 0)}{\Delta y} \\
& \frac{\partial V}{\partial z}(x, y, 0)=0
\end{aligned}
$$

Efetuando pequenos deslocamentos laterais $\Delta x$ ou $\Delta y$ na posição $(x, y)$ do eletrodo 2 , pode-se medir os gradientes $\partial V / \partial x$ ou $\partial V / \partial y$.

As relações entre o gradiente de potencial $\nabla V$, o campo elétrico $\mathbf{E}$ e a densidade de corrente $\mathbf{J}$ num material condutor são descritas em (Hayt, 1958) e (Bowler, 2006). A partir do campo elétrico superficial $\mathbf{E}$, a densidade de corrente $\mathbf{J}$ associada, logo abaixo da superfície da placa, pode ser calculada usando (5). Sendo $\sigma$ a condutividade da placa metálica.

$$
\mathbf{J}=\sigma \mathbf{E}
$$

A partir de (3) e (5), resulta (6), que permite calcular a densidade de corrente $\mathbf{J}$ a partir do gradiente de potencial superficial $\nabla V$.

$$
\mathbf{J}=-\sigma \nabla V=-\sigma\left[\frac{\partial V}{\partial x}(x, y, 0), \frac{\partial V}{\partial y}(x, y, 0), 0\right]
$$

Cabe destacar que (6) permite determinar as componentes $J_{x}$ e $J_{y}$ da densidade de corrente $\mathbf{J}$.

\subsection{A Detecção de Defeitos Através do PD}

A detecção de defeitos por PD tem sido referenciada em alguns trabalhos (Sposito, 2009), (Sposito et al., 2010). A Figura 2 apresenta um desenho em corte da placa, mostrada na Figura 1, onde o plano de corte passa pela linha $a a^{\prime}$, sendo mostrados os 4 eletrodos. A figura apresenta duas configurações de placa, sem defeito (a) e com defeito (b). O defeito consiste num trecho vazio, simulando a perda localizada de material na placa, com seção quadrada, de largura $a$, altura $t$ e alinhado com a face da placa oposta aos eletrodos (onde $z=-T$ ). O comprimento lateral do defeito $a$ é bem menor que a largura e comprimento da placa, $(a<<A, a<<B)$ na Figura 1. Comparando as condições (a) e (b) da Figura 2, na condição (b), devido ao defeito, a seção reta de passagem da corrente $A$ é menor em $x=0$, resultando no aumento da densidade de corrente, conforme (1) e também no módulo do gradiente $|\partial V / \partial x|$ superficial, conforme (6). Na região condutora, entre a superfície da placa $(x=0, y=0, z=0)$ e o topo do defeito, em $(x=0, y=0, z=-T+t)$, a densidade $\mathbf{J}$ aumenta, resultando em $\mathbf{J}_{D}>\mathbf{J}_{S}$, conforme a Figura 2, sendo $I$ a corrente constante aplicada nas condições (a) e (b).

Conforme (6), variações na densidade de corrente próximas à superfície influenciam o gradiente de potencial superficial, que podem ser medidos com o eletrodo 2,
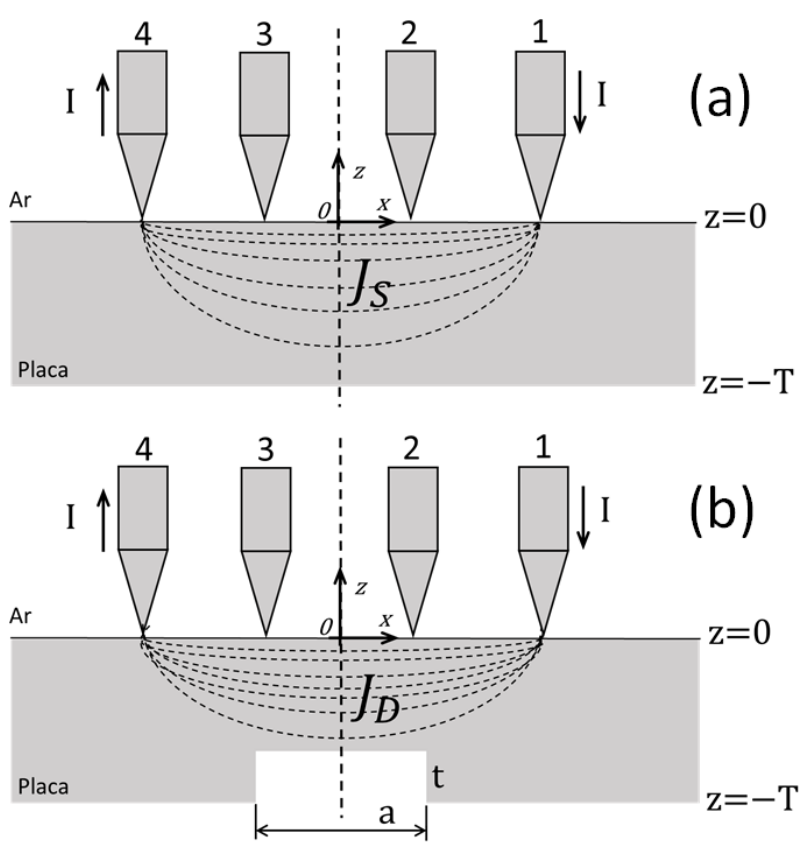

Figura 2. Densidades de corrente $\mathbf{J}$ em $x=0$ : (a) Placa sem defeito, densidade $\mathbf{J}_{S}$. (b) Placa com defeito (largura $a$ e profundidade $t$ ), densidade $\mathbf{J}_{D}$.

conforme exposto, realizando-se pequenos deslocamentos $\Delta x$ e $\Delta y$ laterais. Além disso, efetuando uma extensa varredura na superfície da placa, utilizando o eletrodo 2 , além de permitir calcular os gradientes de potencial, é possível determinar a localização e delimitar a geometria de defeitos na placa. Os defeitos internos ou superficiais à placa, tais como trincas, perdas materiais por corrosão e outros, geram descontinuidades elétricas e variações na área de condução, gerando desvios na direção e no módulo (aumento) da densidade de corrente $\mathbf{J}$ no entorno dos defeitos. As variações de densidade de corrente $\mathbf{J}$ podem ser detectadas na superfície da placa, a partir de suas componentes $J_{x}$ e $J_{y}$, calculadas a partir de (4) e (6). Os defeitos também pode ser detectados analisando diretamente as variações no gradiente superficial $\nabla V$, calculado com (4).

\section{MODELAGEM POR ELEMENTOS FINITOS}

Devido as dificuldades em obter soluções analíticas para a modelagem elétrica das peças a serem analisadas, com a técnica de PD, a literatura (Sposito, 2009) tem utilizado a modelagem FEM em aplicações similares à deste trabalho.

Foram construídas placas e realizada a modelagem FEM das placas com e sem defeito. As características e condições de injeção de corrente são apresentadas na Tabela 1. As coordenadas seguem o padrão da Figura 1, com a referência $(0,0,0)$ no centro da placa. O defeito foi usinado no centro da face inferior de uma das placas. A modelagem FEM das placas foi realizada com o software COMSOL ${ }^{R}$.

\subsection{Resultados da modelagem por elementos finitos}

As Figuras 3 e 4 apresentam a vista superior do trecho central $(-7 \mathrm{~cm}<x<7 \mathrm{~cm}$ e $-4 \mathrm{~cm}<y<4 \mathrm{~cm})$ das placas sem e com defeito respectivamente, mostrando as 
Tabela 1. Placas construídas e modeladas FEM.
- Material da placa: aço inox AISI-304
- Dimensões da placa (quadrada): $A=B=300 \mathrm{~mm}$
- Espessura da placa: $T=10 \mathrm{~mm}$
- Dimensões do defeito ${ }^{(1)}$ (quadrado): $a=b=40 \mathrm{~mm}$
- Profundidade do defeito ${ }^{(1)}: t=3 \mathrm{~mm}$
- Corrente injetada: 10A (CC)
- Posição dos eletrodos de injeção (1-4): $x_{1}=120 \mathrm{~mm}, x_{4}=-120 \mathrm{~mm}$
Nota (1): apenas na placa defeituosa

curvas de densidade de corrente $\mathbf{J}$ nas placas, obtidas com a FEM. Para fins comparativos, o quadrado indica a localização das bordas do defeito, existente na placa defeituosa. Comparando as duas figuras, pode-se notar uma perturbação nos vetores densidade de corrente $\mathbf{J}$, em direção e módulo, na área do defeito.



Figura 3. Densidade de corrente (J) na placa sem defeito, sendo destacada a região onde estará o defeito.

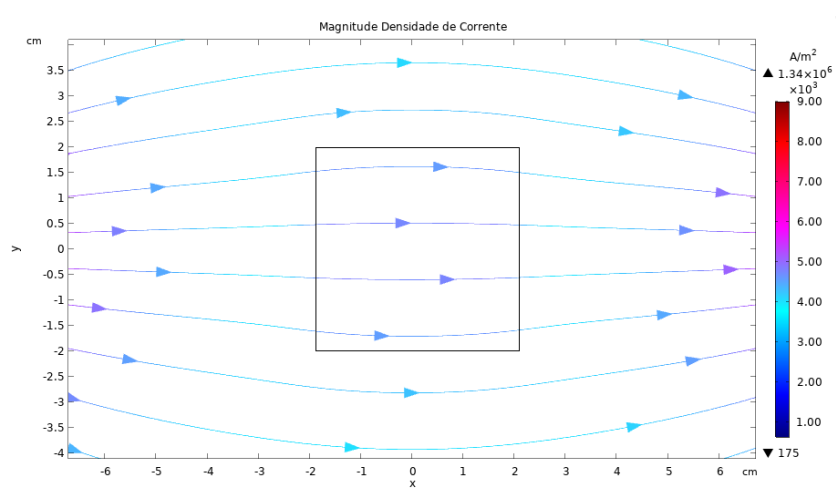

Figura 4. Densidade de corrente (J) na placa com defeito, sendo destacada a região onde está o defeito, bordas em $x= \pm 2 \mathrm{~cm}$ e $y= \pm 2 \mathrm{~cm}$.

Para avaliar os gradientes de potencial de superfície, relacionados à densidade de corrente conforme (6), as Figuras 5 e 6 mostram as curvas isogradientes de potencial (módulo de isogradientes), nas placas sem e com defeito respectivamente. São perceptíveis o elevado gradiente no entorno de cada eletrodo de injeção de corrente 1 e 4. Na Figura 6, em sua região central, onde se localiza o defeito, circunscrito por suas bordas em $x= \pm 2 \mathrm{~cm}$ e $y= \pm 2 \mathrm{~cm}$, os gradientes de potencial mais intensos destacam a localização e a geometria do defeito, descrito na Tabela 1.

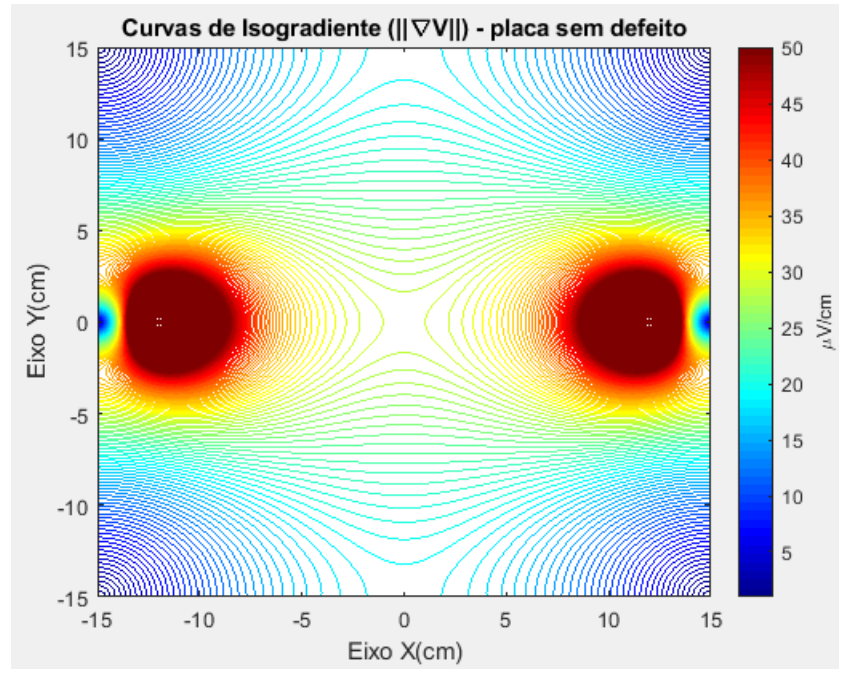

Figura 5. Curvas de isogradiente de potencial superficial, $\|\nabla V\|$, na placa sem defeito.

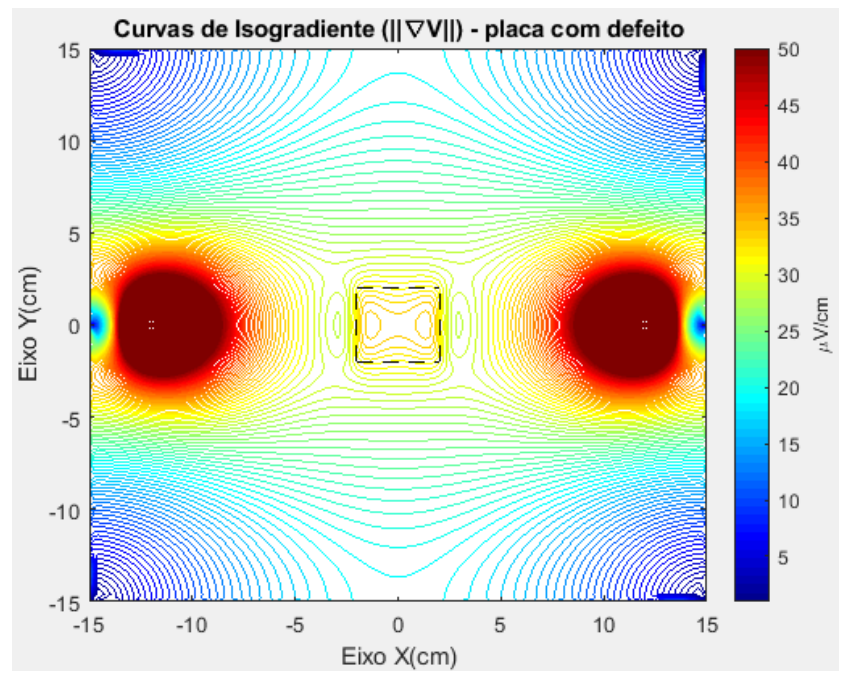

Figura 6. Curvas de isogradiente de potencial superficial, $\|\nabla V\|$, na placa com defeito, cujas bordas (linha tracejada) estão em $x= \pm 2 \mathrm{~cm}$ e $y= \pm 2 \mathrm{~cm}$

Para ressaltar a detecção através de gradientes de potencial de superfície, as Figuras 7 e 8 apresentam os gradientes de potencial (módulo do gradiente, $-\|\nabla V\|$ ) no trecho mais central $(-4 \mathrm{~cm}<x<4 \mathrm{~cm}$ e $-4 \mathrm{~cm}<y<4 \mathrm{~cm})$, nas placas sem e com defeito respectivamente. Os gráficos apresentam o gradiente com sinal invertido, $-\nabla V$, visando tornar o acréscimo na indicação do gráfico compatível com o aumento na densidade de corrente $\mathbf{J}$, conforme (6). A elevação dos gradientes no centro da placa da Figura 6 destacam a localização e a geometria do defeito, descrita na Tabela 1 . No centro do defeito e na placa defeituosa, em $(0,0,0)$, o gradiente $\partial V / \partial x=-33,22 \mu V / \mathrm{cm}$ foi quase o mesmo medido nos testes de laboratório, como será mostrado.

A Figura 9 apresenta os gradientes de potencial, $\partial V / \partial x$, nas placas sem e com defeito. Obtidos da FEM ao longo do eixo $x$, coordenadas $(x, 0,0)$, onde $-4 \mathrm{~cm}<x<4 \mathrm{~cm}$. Comparando as placas sem e com defeito, o gradiente de potencial apresenta inflexões em $x= \pm 2 \mathrm{~cm}$, compatíveis 


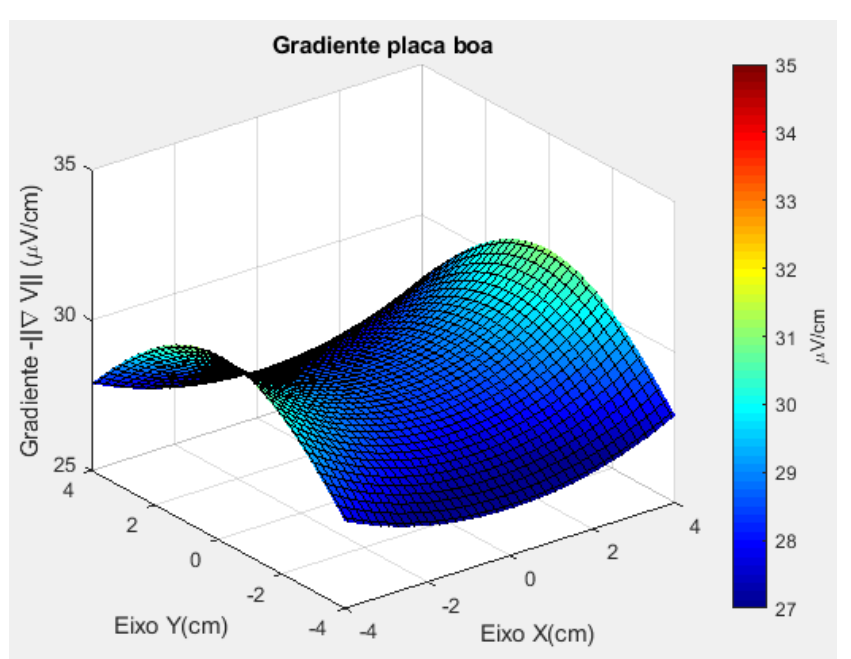

Figura 7. Curva de gradiente de potencial superficial, $-\|\nabla V\|$, na placa sem defeito.

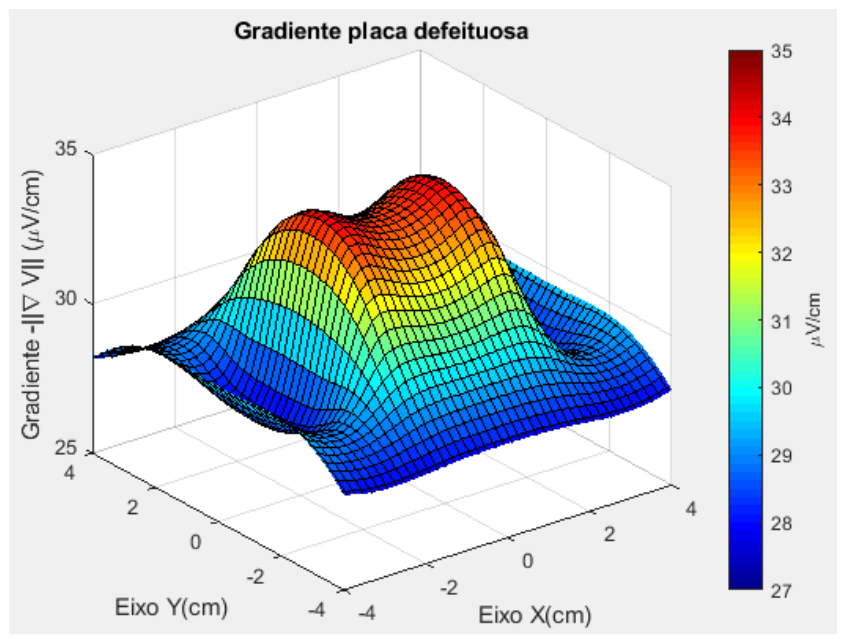

Figura 8. Curva de gradiente de potencial superficial, $-\|\nabla V\|$, na placa com defeito, cujas bordas são $x=$ $\pm 2 \mathrm{~cm}$ e $y= \pm 2 \mathrm{~cm}$

com a geometria do defeito, detalhado na Tabela 1 e conforme (6) e (1).

Além do gradiente de potencial, a detecção de defeitos pode ser realizada através da comparação entre as placas boa e defeituosa. A Figura 10 apresenta o gráfico com a diferença entre os potenciais de superfície das placas sem e com defeito, ao longo do eixo $x$ de -11 a $+11 \mathrm{~cm}$. As linhas verticais indicam as bordas do defeito. As transições do gráfico estão compatíveis com as bordas do defeito, em $x= \pm 2 \mathrm{~cm}$. Esse tipo de comparação se baseia no conceito de mudança na assinatura elétrica da placa boa, visando detectar mudanças, que visem a detecção e a monitoração da evolução de defeitos. Bem como, na determinação da imagem elétrica de defeitos internos.

\section{TESTES EM LABORATÓRIO}

Foram construídas placas conforme a Tabela 1, submetidas à testes de medição de potenciais superficiais. Foi utilizado o arranjo básico de eletrodos da Figura 1. O sistema de

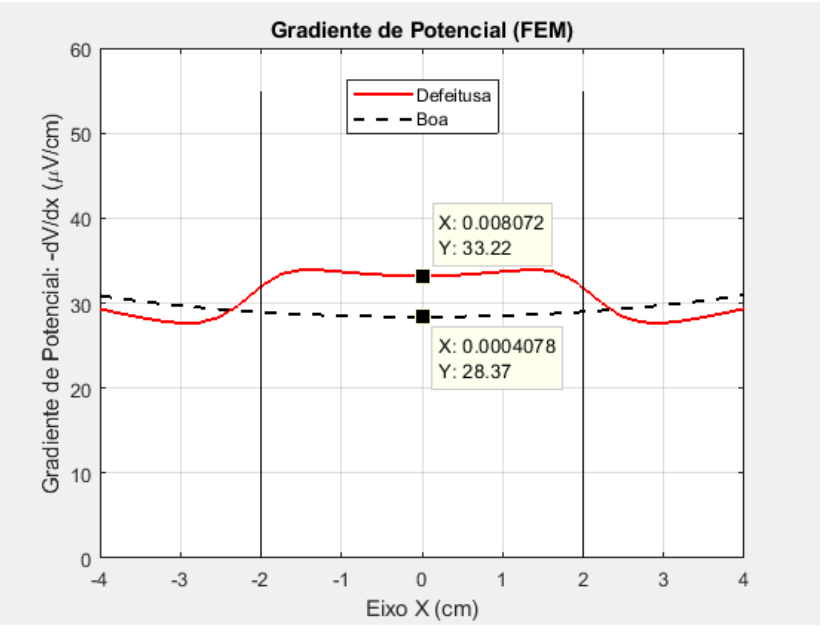

Figura 9. Gradiente de potencial superficial, $-\partial V / \partial x$, nas placas sem e com defeito. Ao longo do eixo $x$, coordenadas $(x, 0,0)$, onde $-4 \mathrm{~cm}<x<4 \mathrm{~cm}$. As linhas verticais indicam as bordas do defeito.

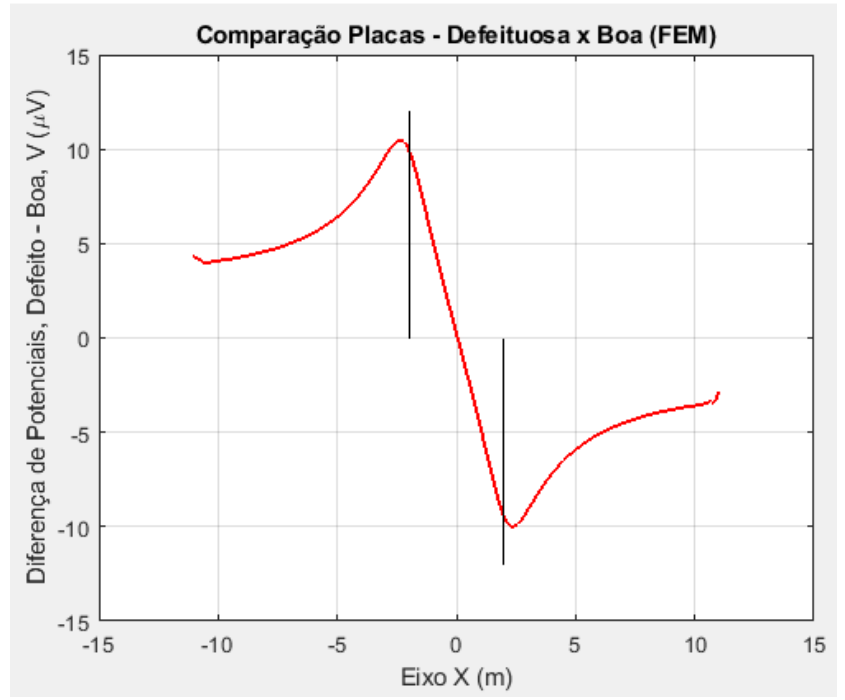

Figura 10. Detecção do defeito por diferença entre potenciais de superfície, nas placas sem e com defeito, obtidas ao longo do eixo $x$, coordenadas $(x, 0,0)$, onde $-11 \mathrm{~cm}<x<11 \mathrm{~cm}$. As linhas verticais indicam as bordas do defeito.

medição de potenciais, que também efetua a injeção de correntes, consiste dos seguintes equipamentos.

- Multímetro digital Agilent 3458A de 8,5 dígitos.

- Fonte HP 6268B, de 0-40V x 0-30A.

- Módulo de aquisição NI USB-6210.

- Módulo de controle de motor de passo, com driver de 4 eixos, baseado em Arduino Mega.

- Estação de controle e medição (Notebook), com software desenvolvido em LABView ${ }^{\circledR}$.

- Sistema de posicionamento de eletrodos móveis.

A estação de controle e medição realiza a varredura da superfície com o eletrodo móvel, comandando sequencialmente o motor de passo e em efetuando medições automáticas através do multímetro de 8,5 dígitos, a precisão do multímetro foi essencial na medição dos potenciais, da ordem de microvolts, com a precisão adequada. 
As medições de potencial realizadas e os valores obtidos por modelagem FEM são apresentados nos gráficos da Figura 11, para comparação. Os valores estão ao longo do eixo $x$, coordenadas $(x, 0,0)$, onde $-4 \mathrm{~cm}<x<4 \mathrm{~cm}$. As linhas verticais indicam as bordas do defeito. O erro médio quadrático (RMS), entre os valores medidos e calculados é de $2,1 \mu V$, espera-se que esse desvio seja ainda menor, pois não foi considerada a diferença de temperaturas, ambiente de medição e do material, na modelagem FEM. Bem como na compensação das tensões termoelétricas, devido ao uso de materiais distintos, nos eletrodos de medição e da placa e possíveis diferenças de temperatura entre os eletrodos de medição 2 e 3, que será compensada com a inversão da polaridade da fonte de corrente e medições de potencial nas duas condições.

O gradiente de potencial $\partial V / \partial x$, no ponto central $(0,0,0)$ na placa com defeito, apresentado na Figura 8 (placa defeituosa), apresentou uma diferença inferior a 1\%, entre a medição no laboratório e o modelo FEM.

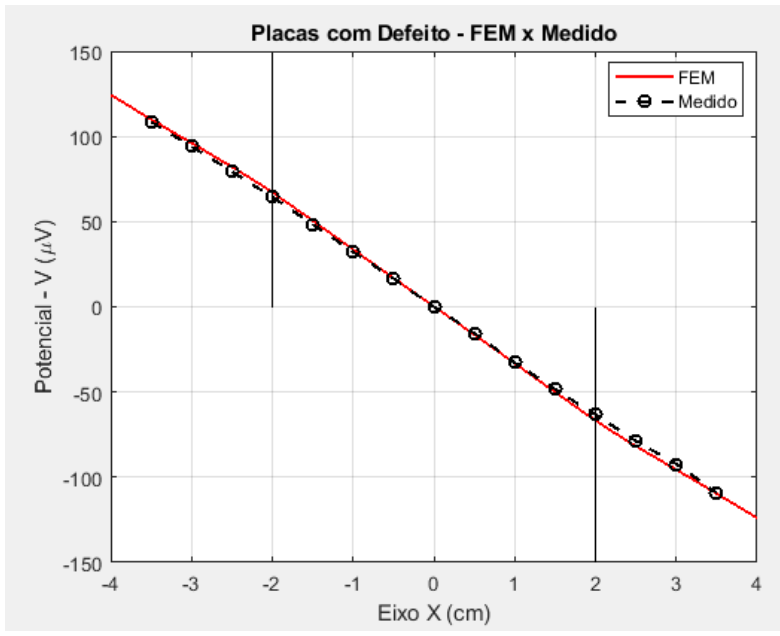

Figura 11. Comparação dos potencias medidos e obtidos por modelagem FEM, na placa defeituosa. As linhas verticais indicam as bordas do defeito.

A Figura 12 apresenta a foto do sistema de medição de potencial, com ênfase na placa, eletrodos de injeção de corrente, sistema de eletrodo móvel, fiação de injeção de corrente (fios azuis ao fundo) e grampos de fixação à chapa.

\section{CONCLUSÃO}

O presente trabalho apresentou a aplicação da técnica PD para a obtenção da imagem elétrica de defeito numa placa de aço inox 304, que poderá ser estendida para outros materiais metálicos utilizados na indústria. Visando a sua futura aplicação, para a detecção de defeitos em tubulações e equipamentos industriais, bem como na monitoração da deterioração desses sistemas em tempo real. Os gráficos e imagens elétricas obtidas, basearam-se em medições de potencial elétrico superficiais e modelos FEM, indicam a viabilidade da técnica apresentada. Também foi mostrada a aplicação da um técnica de detecção utilizando a diferença de potenciais de superfície, entre uma placa boa e uma placa defeituosa. Que indicou a viabilidade deste conceito, de mudança na assinatura elétrica da placa, para



Figura 12. Foto do sistema de medição de potencial, com ênfase na placa, eletrodos de injeção de corrente e sistema de eletrodo móvel.

a detecção e a monitoração da evolução de defeitos, bem como, para melhorias na imagem elétrica de defeitos internos. Em trabalhos futuros, a aplicação de técnicas heurísticas pode melhorar os resultados obtidos, no diagnóstico de defeitos, na monitoração em tempo real da deterioração de instalações e na geração de imagens elétricas de defeitos.

\section{REFERÊNCIAS}

A. R. Al-Shamari, A. W. Al-Mithin, S. Al-Sulaiman and J. Amer, Pipeline integrity management through internal corrosion monitoring. NACE Corrosion, San Antonio, Texas, 2014.

G. Sposito, P. Cawley and P. Nagy, Potential drop mapping for the monitoring of corrosion or erosion. NDTEE International, pages 394-402, March 2010.

G. Sposito, Advances in potential drop techniques for non-destructive testing. PhD Thesis, Imperial College, London, 2009.

L. Yang, Techniques for corrosion monitoring. Houston, Texas. Woodhead Publishing, 2008.

N. Bowler, Theory of Four-Point Direct-Current Potential Drop Measurements on a Metal Plate. Research in Nondestructive Evaluation, volume 17, pages 29-48, 2006.

NITG390, Techniques for monitoring corrosion and related parameters in field applications. NACE International, Houston, Texas. Publication 3T199, 2013.

P. R. Roberge, Corrosion inspections and monitoring. Kingston/Ontario, John Wiley \& Sons, 2007.

V. G. Silva, G. L. Vaz, P. A. Ferreira, A. Ramus and N. L d'Almeida, Evaluation of non-intrusive systems for internal corrosion monitoring. Rio Pipeline Conference Rio de Janeiro, 2017.

V. G. Silva , G. R. V. Pinheiro and J. A. C. P. Gomes, Técnicas de Queda de Potencial para Monitoração de Corrosão Interna. COTEQ-2019:Conferência sobre Tecnologia e Equipamentos, IBP, Rio de Janeiro, 2019.

W. H. Hayt, Engineering Eletromagnetics. McGraw-Hill, 2nd Ed., 1958. 